\title{
Value of abdominal radiograph in the follow-up of spinal cord injury patients
}

Spinal Cord (2006) 44, 63. doi:10.1038/sj.sc.3101766; published online 19 July 2005

Tins et $a l,{ }^{1}$ in an article published recently in Spinal Cord, state that the KUB radiograph does not seem justified in the routine follow-up of the urinary tract in spinal injury patients. Patients, who had sustained spinal cord injury, attend the spinal unit for global check-up; these patients do not come to the spinal unit for an assessment of the urinary tract alone. X-ray of the KUB or abdominal radiograph represents one of the many tests of surveillance carried out during a follow-up visit to the spinal injuries centre. We found the abdominal radiograph to provide valuable information about bowel gas pattern, faecal loading, redundant sigmoid loop, dilated large bowel especially the caecum, presence of spinal curvature, vascular calcification, gall stones, pancreatic calcification, Charcot's spine, and status of hip joints in addition to the presence of any radioopaque calculi in the urinary tract.

Dysfunction of bowels in spinal cord injury patients causes considerable morbidity and, rarely, mortality as well. Redundant sigmoid bowel was noted during a routine follow-up of a patient with C-4 tetraplegia. About 6 months later, this patient developed sigmoid volvulus and succumbed to respiratory complications in a local district general hospital. Since then we tend to be proactive in treating patients with redundant sigmoid colon. Similarly, we consider total colectomy as one of the options for managing globally dilated large bowel in a tetraplegic patient, in whom the distended colon causes compromise to lung function because of the sheer volume occupied by the large bowel, which has gaseous distension and faecal loading. Abdominal distension and consequent upward displacement of domes of the diaphragm in tetraplegic subjects lead to a significant reduction in lung volumes, which often results in desaturation (hypoxaemia).

In conclusion, we believe that the abdominal radiograph provides valuable information, especially regarding the status of the neuropathic bowel, and should not be omitted during annual follow-up visits of spinal cord injury patients.

S Vaidyanathan ${ }^{1}$, BM Soni ${ }^{1}$ and PL Hughes ${ }^{2}$

${ }^{1}$ North-West Regional Spinal Injuries Centre, District General Hospital, Southport PR8 6PN, UK; ${ }^{2}$ Department of Radiology, District General Hospital, Southport PR8 6PN, UK

\section{References}

1 Tins B, Teo HG, Popuri R, Cassar-Pullicino V, Tyrrell P. Follow-up imaging of the urinary tract in spinal injury patients: is a KUB necessary with every ultrasound? Spinal Cord 2005; 43: 219-222. 